\title{
Generalization by Optimization in Menger Probabilistic Normed Spaces with Application on Portfolio in Insurance
}

Weber $\mathbf{G W}^{*}$ and Solatikia F

Institute of Applied Mathematics, Middle East Technical University, Ankara, Turkey

\begin{abstract}
The purpose of this paper is to show that the category of normed spaces can be embedded in the category of Menger probabilistic normed spaces, and that $\mathrm{C}(\Omega)$ is probabilistic normable, whereas it is not normable in the classical case, when $\Omega$ is an open subset of $\mathrm{R}^{n}$. So, the spectrum of the category of Menger probabilistic normed spaces is broader than the category of classical normed spaces. Therefore, it can be a meaningful replacement in some model of security markets. As our model is suitably generalized, that fact can help us to adapt and improve within natural problems of finance, especially, in portfolio optimization of insurance.
\end{abstract}

Keywords: Menger probabilistic; Normed spaces; Fuzzy normed spaces; Portfolio optimization; Finance; Insurance

\section{Introduction}

It is well known that the theory of Menger probabilistic normed spaces is a new frontier branch between probability theory and functional analysis and has an important background, which contains the common normed space as a special case and it is used to study the problem of best approximation in statistics. The first idea of K. Menger in 1942 [1] was to use distribution functions instead of nonnegative real numbers as values of the metric. It is also known that the theory of Menger probabilistic normed spaces is a generalization of fuzzy normed spaces, introduced by Felbin [2], and T. Bag and S.K. Samanta [3], which the latter has already been studied by the authors in $[4,5]$. By applying the method of neighborhood and introducing the probabilistic semi norm we mainly discuss the topological vector space structure of Menger probabilistic normed spaces and show that a Menger probabilistic normed space is also a topological vector space in the sense of classical analysis. So, most important theorems and results, for instance, Hahn-Banach Theorem, Open Mapping Theorem and Closed Graph Theorem, can be easily established in Menger probabilistic normed spaces, in general. As a new result, we show that $\mathrm{C}(\Omega)$, the set of all real-valued continuous functions on an open set $\Omega$ is a Menger probabilistic normed space, while it is not classically normable. By addressing an open set $\Omega$, we go beyond the case of a compact set which is the classical case in literature. So, if we replace the normed space by the Meneger probabilistic normed space $C(\Omega)$, then we get an important result in Menger probabilistic normed spaces, while the same statement does not hold true in classical analysis.

Definition 1: A triplet $\left(X, N,{ }^{*}\right)$ is called a Menger probabilistic normed space (briefly, Menger PN-space) if $X$ is a real vector space, $N$ is a mapping from $X$ into the set of all distance distribution functions, denoted by $\Delta^{+}$(for $x \in X$ there is a distribution function $N(x)$ and $N(x, t):=(N(x))$ $(t)$ is the value of $N(x)$ at $t \in R)$, and $\in$ is a so-called $t$-norm, satisfying the subsequent conditions: $(P N-1) N(x, t)=\in 0$ for some $\in 0>0$ and for all $t>0$ iff $x=0$. $(P N-2) N(c x, t)=N\left(x, \frac{t}{|c|}\right)$ for all $c \in R n \backslash\{0\}$. (PN-3) $N$ $(x+y ; s+t) \geq N(x, s){ }^{*} N(y, t)$ for all $x, y \in X$, and for all $s, t>0$. We call the mapping $\mathrm{x} \mapsto \mathrm{N}(\mathrm{x}) \mathrm{a}^{*}$-norm or $\mathrm{a}^{*}$-probabilistic norm on X. If ${ }^{\star}=$ min, we will denote $\left(X, N,{ }^{\star}\right)$ by $(X, N)$.

Felbin and Samanta showed that any classical real normed space can be considered as fuzzy normed linear spaces in $[2,3]$. It was known that the theory of Menger probabilistic normed spaces is a generalization of fuzzy normed spaces; that special case has already been studied in $[4,5]$. In the following, we also present an example of a Menger probabilistic normed space which is not a real normed space in the classical sense.

\section{Main Results}

The classical theory of subspaces $\mathrm{C} \Omega$ whose domain $\Omega$ is any compact Haus-dorff topological space, as well as elements from the theory of finance are very useful, towards the construction of a powerful and efficient package for the translation of mathematical problems from economics into the language of computing. In particular, Polyrakis [6] provided a solution to the problem of whether a finite collection of linearly independent positive functions in $\mathrm{C}[\mathrm{a}, \mathrm{b}]$, i.e., $\Omega=[\mathrm{a}, \mathrm{b}]$ generates a subspace. The book [7] presents theory, methodology and applications of Itó calculus to solve problems in modern finance. In [6], the author studied this minimization problem under the general assumption that $\mathrm{X}$ is contained in a finite-dimensional minimal subspace. The model of security markets studied in [8] became extended over two periods, period 0 and period 1, and is as follows: Suppose that there are $n$ securities labeled by the natural numbers $1,2, \ldots, n$, acquired in period 0 . Securities are described by their payoffs at date 1 . The payoff of the ith security is in general a positive element $x_{i}$ of an ordered space $\mathrm{E}$ which is called the payoff space. It is also assumed that the payoffs $\mathrm{x}_{1}$, $\mathrm{x}_{2} \ldots \mathrm{x}_{\mathrm{n}}$ are linearly independent vectors of $E$. In [8], it was supposed that $\mathrm{E}$ is the space of real-valued continuous functions $C(\Omega)$ defined on a compact Hausdorff topological space ; therefore, the payoff vectors xi are linearly independent, positive elements of $C(\Omega)$. The elements of the set express all the "possible states of the world" during period 1 , and the value $x_{i}(t)$ is the payoff of the security $i$ in the time $t$. Therefore, function $x_{i}(1)$ is the profile of the $i$ th security at period

1. A portfolio is a vector $\alpha=\left(\alpha_{1}, \alpha_{2} \ldots \alpha_{n}\right)$ of $\mathrm{R}^{n}$, where $\alpha_{i}$ is the number

${ }^{*}$ Corresponding author: G.W. Weber, Institute of Applied Mathematics, Middle East Technical University, Ankara, Turkey, E-mail: gweber@metu.edu.tr

Received April 25, 2013; Accepted April 25, 2013; Published April 29, 2013

Citation: Weber GW, Solatikia F (2013) Generalization by Optimization in Menger Probabilistic Normed Spaces with Application on Portfolio in Insurance. J App Computat Math 2: e131. doi:10.4172/2168-9679.1000e131

Copyright: @ 2013 Weber GW, et al. This is an open-access article distributed under the terms of the Creative Commons Attribution License, which permits unrestricted use, distribution, and reproduction in any medium, provided the original author and source are credited. 
Citation: Weber GW, Solatikia F (2013) Generalization by Optimization in Menger Probabilistic Normed Spaces with Application on Portfolio in Insurance. J Appl Computat Math 2: e131. doi:10.4172/2168-9679.1000e131

Page 2 of 2

of shares of the ith security. The space $\mathrm{R}^{n}$ is called the portfolio space. The payoff of portfolio $\alpha(\alpha \in \Omega)$ is $\mathrm{R}(\alpha):=\sum_{i=1}^{n} \alpha_{i} x_{i}(1)$; then, $\mathrm{R} \in \mathrm{C}(\Omega)$. Finally, the author showed that if the payoff space $\mathrm{X}$ is contained in a finite-dimensional minimal subspace $\mathrm{Y}$ of $\mathrm{C}(\Omega)$ and the component-wise sum of the payoff vectors $x_{i}$ is strictly positive, then a minimum-cost insurance of the portfolio $\alpha$ at the floor $\mathrm{k}$ and in the price $q$ exists and it is determined by solving the problem of minimization of a positive linear functional under a finite number of linear inequalities.

By the following theorem, prepared for a Menger probabilistic normed space, but not generally true for a real normed space in the classical sense, we know that the spectrum of the category of Menger probabilistic normed linear spaces is broader than the category of fuzzy normed spaces and normed spaces. That is why the study of Menger probabilistic normed linear spaces has a great importance.

Theorem 1: Let $\Omega$ be a nonempty open set in some Euclidean space $R n$. The linear space $C(\Omega)$ is a Menger probabilistic normed space in general. But $C(\Omega)$ is not normable in classical analysis. Also, it is a Menger probabilistic Banach algebra in general.

Remark 1: In this paper, by proving that the space $(C(\Omega), N)$ is a Menger probabilistic normed space, we could assume any nonempty open set $\Omega$ in some Euclidean space Rn instead of a compact Hausdorff topological space in the above theory for a computational method of solving our particular minimization or maximization problem. Therefore, the new method makes our domain extended, which can help us to adapt and apply in emerging problems of financial mathematics and actuarial sciences, especially, in portfolio optimization. Also, indicating that the category of Menger probabilistic normed spaces is, isomorphically regarded, a subcategory of the category of topological vector spaces, we will become able to use this space in order to get the best approximation in calculating the minimum cost insured portfolio.

\section{References}

1. Menger K (1942) Statistical metrics. Proc Nat Acad Sci USA 28: 5357.

2. Felbin C (1992) Finite dimensional fuzzy normed linear spaces. Fuzzy Sets Syst 48: 239-248.

3. Bag T, Samanta SKN (2003) Finite dimensional fuzzy normed linear spaces Fuzzy Math 11: 687-705.

4. Sadeqi I, SolatiKia F (2009) Fuzzy normed linear space and its topologica structure. Chaos, Solitons and Fractals, 40: 2576-2589.

5. Sadeqi I, SolatiKia F (2010) The category of fuzzy normed linear spaces. Fuzzy Math 18: 733-742.

6. Polyrakis IA (1996) Finite-dimensional lattice-subspaces of $C(\Omega)$ and curves of Rn. Transactions of the American Mathematical Society 348: 2793- 2810.

7. Korn R, Korn E (2001) Option Pricing and Portfolio Optimization. American Mathematical Soc 31.

8. Polyrakis IA (2003) Linear Optimization in $C(\Omega)$ and Portfolio Insurance Optimization 52: 221-239. 